\title{
A COVID-19 patient with seven consecutive false-negative rRT-PCR results from sputum specimens
}

\author{
Cong-Ying Song ${ }^{1,2} \cdot$ Da-Gan Yang ${ }^{3} \cdot$ Yuan-Qiang $\mathrm{Lu}^{1,2}$
}

Received: 11 May 2020 / Accepted: 26 June 2020 / Published online: 2 July 2020

(c) Società Italiana di Medicina Interna (SIMI) 2020

Keywords COVID-19 $\cdot$ False-negative $\cdot$ rRT-PCR $\cdot$ SARS-CoV-2

Dear Editor,

The Coronavirus Disease 2019 (COVID-19) pandemic has become a great threat to public health [1]. Nucleic acid detection for severe acute respiratory syndrome coronavirus 2 (SARS-CoV-2) by real-time reverse-transcriptase polymerase chain reaction (rRT-PCR) is regarded as the gold standard method for COVID-19 diagnosis, yet several studies have reported that rRT-PCR yields false-negative results $[2,3]$. Here, we report a case of severe COVID-19 with initial seven consecutive false-negative rRT-PCR results in the First Affiliated Hospital, School of Medicine, Zhejiang University.

A 30-year-old man visited the fever clinic of our hospital on January 22, 2020, presenting with fever (peak body temperature: $37.9{ }^{\circ} \mathrm{C}$ ), fatigue, chills, dizziness, and chest tightness for 3 days. He confirmed of having come in close contact with a colleague with SARS-CoV-2 infection. Computed tomography (CT) showed patchy dense shadows with

Yuan-Qiang Lu

luyuanqiang@zju.edu.cn

Cong-Ying Song

21818005@zju.edu.cn

Da-Gan Yang

yangdagan@zju.edu.cn

1 Department of Emergency Medicine, The First Affiliated Hospital, School of Medicine, Zhejiang University, Hangzhou 310003, People's Republic of China

2 Zhejiang Provincial Key Laboratory for Diagnosis and Treatment of Aging and Physic-Chemical Injury Diseases, The First Affiliated Hospital, School of Medicine, Zhejiang University, Hangzhou 310003, People's Republic of China

3 Department of Laboratory Medicine, The First Affiliated Hospital, School of Medicine, Zhejiang University, Hangzhou 310003, People's Republic of China mixed ground-glass opacity in the left lower lung (Fig. 1a). However, his rRT-PCR test results from sputum specimens for SARS-CoV-2 were not positive. Laboratory tests showed that white blood cell $\left(4.2 \times 10^{9} / \mathrm{L}\right)$, neutrophil $\left(2.2 \times 10^{9} / \mathrm{L}\right)$, and lymphocyte $\left(1.36 \times 10^{9} / \mathrm{L}\right)$ counts were within the normal range, while the C-reactive protein (CRP) level was elevated $(15.11 \mathrm{mg} / \mathrm{L})$. Considering the CT features and epidemiological exposure history, he was admitted to our hospital despite the negative rRT-PCR result. During the period of isolated observation and medical care, repeated nucleic acid testing of sputum specimens and chest CT were performed. Results of rRT-PCR were continually negative until the eighth test on day 8 after admission (day 11 after illness onset). The development process and disease treatment are summarized in Fig. 2. The progression of pulmonary lesions was observed on CT 3 days before the first positive rRT-PCR result (Fig. 1b). Pulmonary lesions on CT progressed significantly after testing positive for SARS-CoV-2 infection, and this patient developed severe pneumonia (Fig. 1c, d). The lung ultrasound showed features similar to those observed on CT (Fig. 3). After the systematic treatment for COVID-19, the patient's symptoms were relieved, and the progression of lung lesions was halted. Positive rRT-PCR results continued for 4 days, after which nucleic acid detection of sputum and stool samples turned negative.

Between January 20, 2020 and February 5, 2020, a total of 99 confirmed cases of COVID-19 were detected by rRTPCR in our hospital, with five patients having false-negative rRT-PCR results (false-negative rate $=5.05 \%$ ). False-negative nucleic acid detection results could be caused by various reasons, including the sample quality, viral load, and stage of disease $[4,5]$. Collecting nasopharyngeal or throat swabs may render patients feeling uncomfortable and cause a risk of infection to healthcare workers. Thus, sputum or saliva samples from deep in the throat were collected for nucleic acid detection in our hospital. In this case, the patient had no 


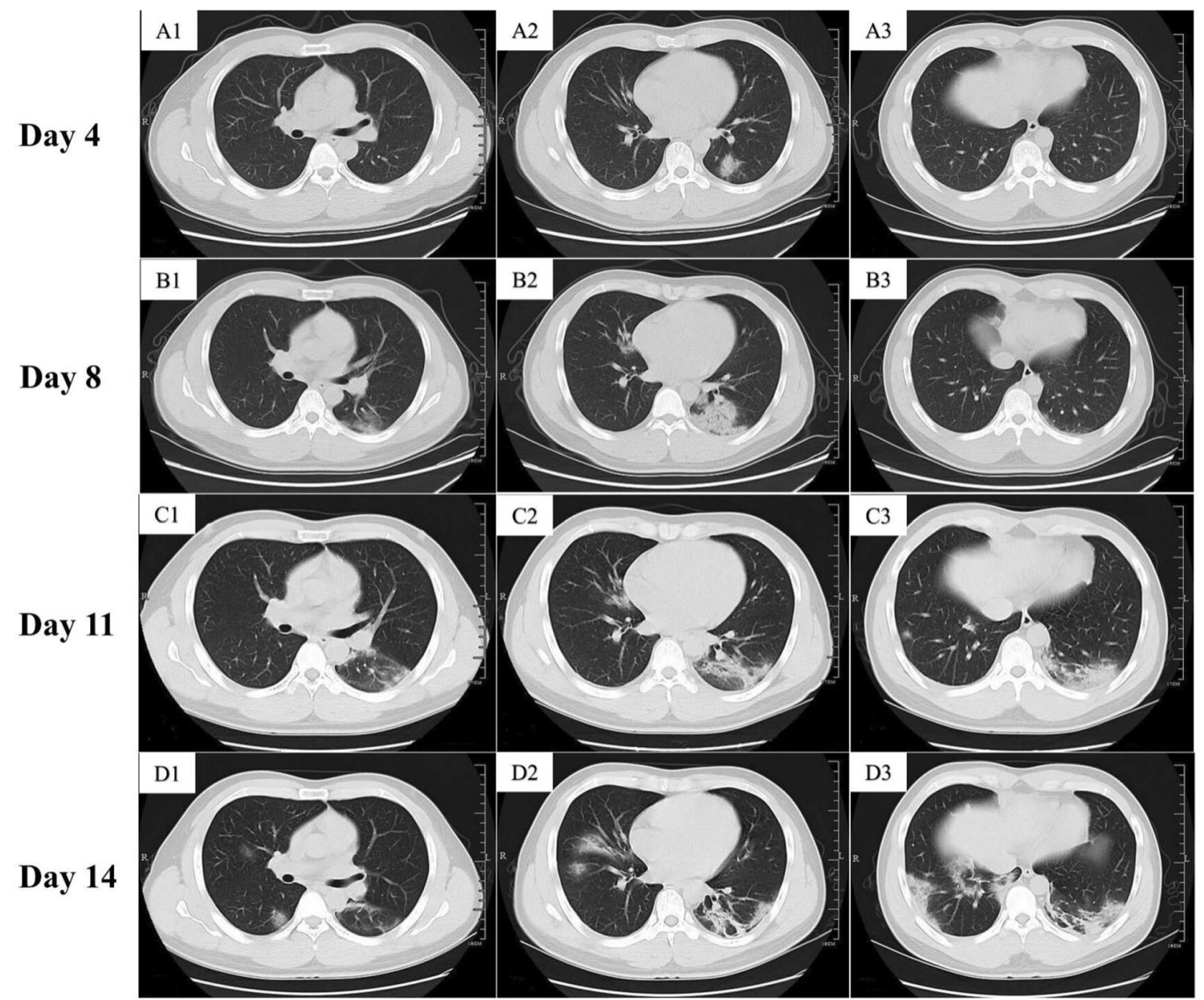

Fig. 1 Imaging features of chest CT. a CT on day 4 (with initial negative rRT-PCR SARS-CoV-2 results) showed patchy dense shadows with mixed ground-glass opacity in the left lower lung. b CT on day 8 (with negative rRT-PCR result) showed patchy and nodular dense shadows in both lower lungs. The scope of pulmonary lesions is enlarged. c CT on day 11 (with initial positive rRT-PCR result) showed multiple patchy and strip-like dense shadows in both lungs.
The lesions have progressed. d CT on day 14 (with positive rRT-PCR result) showed multiple patchy and high dense shadows in both lungs, mainly in the peripheral zone of the lower lobes. The lesions progressed significantly. $C T$ computed tomography, $r R T-P C R$ real-time reverse transcription-polymerase chain reaction, $S A R S-C o V-2$ severe acute respiratory syndrome coronavirus 2

Fever
Fatigue
Cough
Antiviral therapy
Oxygen therapy
Glucocorticoids
CT progression
SARS-CoV-2 RNA positive
Days after illness onset

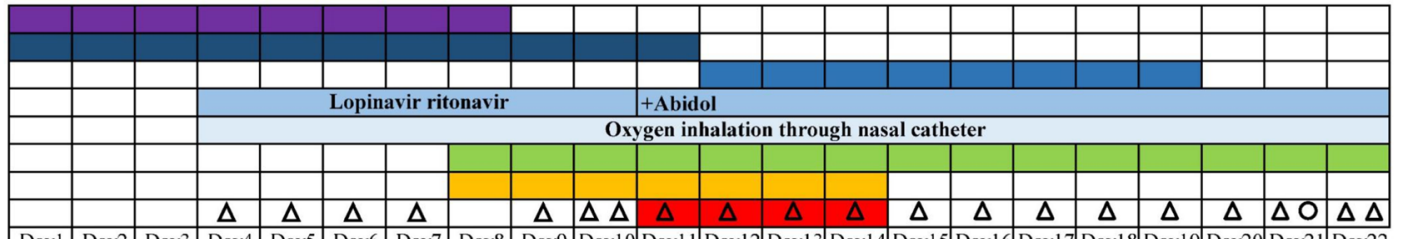

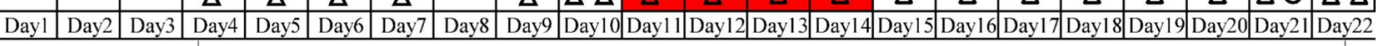

Hospital admission

Discharge

\section{$\Delta$ Respiratory samples}

Stool samples

Fig. 2 Disease development and treatment application. The development process of the disease is shown starting from the illness onset. The patient was admitted to our hospital 3 days after illness onset. rRT-PCR results were consistently negative until 11 days after illness onset (day 8 after admission). The lesions shown in CT progressed before the first positive rRT-PCR result. The virus duration in the respiratory samples was 4 days, during which the pulmonary lesions progressed. After that, patient symptoms were relieved, and the pulmonary lesions were absorbed. $C T$ computed tomography, $r R T-P C R$ real-time reverse transcription-polymerase chain reaction 


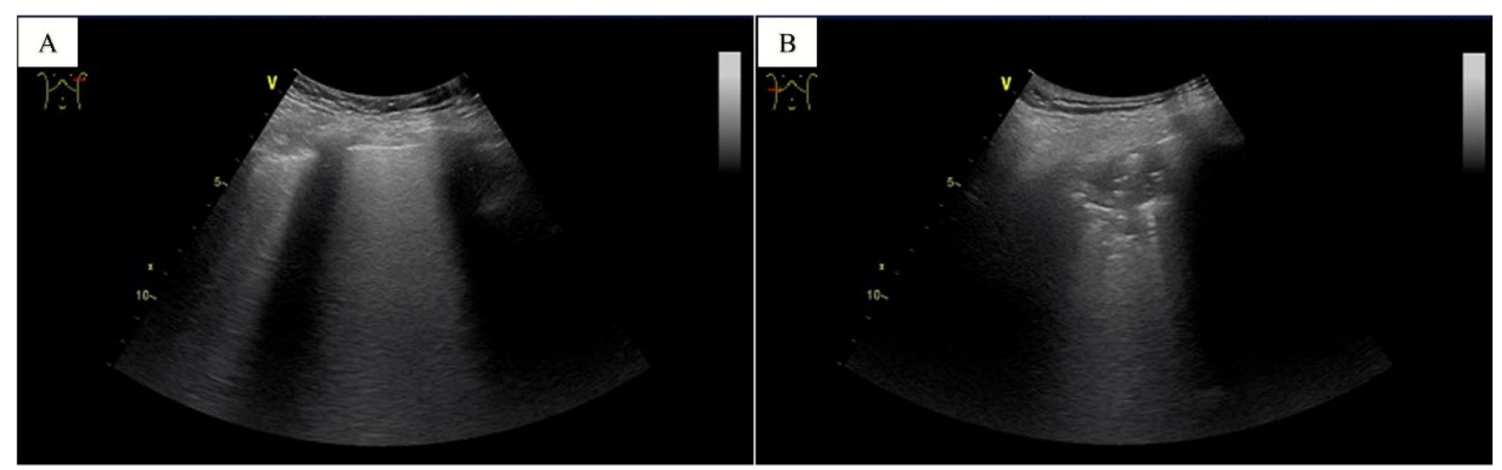

Fig. 3 Imaging features of lung ultrasound. a Lung ultrasound of the left lower lung (day 14) showed confluent B lines, which can be found in interstitial pneumonia. This area showed patchy and striplike dense shadows on CT. b Lung ultrasound of the right lower lung

symptoms of expectoration, so the amount of their sputum samples may have been relatively low with uncertain sample quality. In addition, this patient was in the early stages of COVID-19, which may be related to the lower viral load. We noticed that this patient was young, which means that they most likely had stronger immunity and higher resistance to viral infections, and may have reduced the viral load. In the early stage of the epidemic, clinicians did not realize that the virus could also exist in feces, so this patient did not undergo continuous nucleic acid testing of stool samples. In this case, the evolution of pulmonary lesions on CT was consistent with the severity of the disease, which indicated that CT findings could be used to evaluate the severity and progress of COVID-19 [6]. However, CT can be inconvenient for patients with severe disease, and the process of transferring patients may increase the risk of viral spread. Considering the limitations of CT, researchers tried to use point-of-care ultrasounds to screen COVID-19 patients or to evaluate the progress of pneumonia, which was convenient for radiation-free monitoring at low cost. They found that lung ultrasound yielded findings that were similar to those of CT, and lung ultrasound features were related to the disease stage and severity [7]. Therefore, lung ultrasounds have major clinical implications for the management of SARS$\mathrm{CoV}-2$ infection. In this case, systematic treatments including oxygen therapy, antiviral therapy, and glucocorticoids were performed in time, resulting in a favorable prognosis. It was reported that treatment with glucocorticoids may prolong viral infection in severe COVID-19 patients [8], which may be due to the inhibition of the immune response by steroids, and resulting in the decreased ability of immune cells to clear the virus. This patient received antiviral therapy and low-dose glucocorticoids in the early stage, which may have influenced the viral load. The relationship between steroid treatment and rRT-PCR results should be explored in a welldesigned study. (day 14) showed air bronchograms and shred signs, which are features of lung consolidation. These features were similar to those of CT. $C T$ computed tomography

In summary, young patients who are in the early stages of COVID-19 may be more likely to have false-negative rRT-PCR results. Diagnosis based only on rRT-PCR may miss some inapparent infections due to false-negative results [9]. Epidemic history and image features of chest CT should be considered during COVID-19 diagnosis. Highly suspected patients of SARS-CoV-2 infection with initial false-negative rRT-PCR results still require timely isolation with repeated nucleic acid detection and CT scans. The value of point-of-care ultrasound in disease monitoring cannot be ignored. Further research should investigate the factors causing SARS-CoV-2 false-negative rRT-PCR which results in a larger sample.

Acknowledgements We would like to thank Wen Fang (The First Affiliated Hospital, School of Medicine, Zhejiang University, Hangzhou, China) for providing assistance with language editing.

Author contributions Y-QL conceived and designed the experiments. C-YS collected and analyzed the data. C-YS and D-GY contributed to the writing of the manuscript.

Funding This research was supported by the Foundation of Key Discipline Construction of Zhejiang Province for Traditional Chinese Medicine (No. 2017XK-A36).

\section{Compliance with ethical standards}

Conflict of interest The authors declare no conflict of interest.

Ethical standard This study was approved by the Ethical Committee of the First Affiliated Hospital, School of Medicine, Zhejiang University (code number IIT20200025A).

Research involving human participants or animals This article does not contain any study with animals performed by any of the authors. 
Informed consent Written consent was obtained from each patient or his/her authorized representatives following a full explanation of the study.

\section{References}

1. World Health Organization Coronavirus disease (COVID-2019) situation reports. https://www.who.int/emergencies/diseases/novel -coronavirus-2019/situation-reports/. Accessed 22 Feb 2020

2. Chen Z, Li Y, Wu B, Hou Y, Bao J, Deng X (2020) A patient with COVID-19 presenting a false-negative reverse transcriptase polymerase chain reaction result. Korean J Radiol. https://doi. org/10.3348/kjr.2020.0195

3. Li D, Wang D, Dong J, Wang N, Huang H, Xu H et al (2020) False-negative results of real-time reverse-transcriptase polymerase chain reaction for severe acute respiratory syndrome Coronavirus 2: role of deep-learning-based CT diagnosis and insights from two cases. Korean J Radiol 21(4):505-508. https://doi. org/10.3348/kjr.2020.0146

4. Xie X, Zhong Z, Zhao W, Zheng C, Wang F, Liu J (2020) Chest CT for typical 2019-nCoV pneumonia: relationship to negative RT-PCR testing. Radiology. https://doi.org/10.1148/radiol.20202 00343
5. To KK, Tsang OT, Chik-Yan Yip C, Chan KH, Wu TC, Chan JMC et al (2020) Consistent detection of 2019 novel coronavirus in saliva. Clin Infect Dis. https://doi.org/10.1093/cid/ciaa149

6. Lu YQ (2020) A woman with fever and cough: coronavirus disease 2019. Intern Emerg Med. https://doi.org/10.1007/s1173 9-020-02321-3

7. Peng QY, Wang XT, Zhang LN, Chinese Critical Care Ultrasound Study G (2020) Findings of lung ultrasonography of novel corona virus pneumonia during the 2019-2020 epidemic. Intensive Care Med 46(5):849-850. https://doi.org/10.1007/s00134-020-05996-6

8. Zheng S, Fan J, Yu F, Feng B, Lou B, Zou Q et al (2020) Viral load dynamics and disease severity in patients infected with SARS-CoV-2 in Zhejiang province, China, January-March 2020: retrospective cohort study. BMJ 369:m1443. https://doi. org/10.1136/bmj.m1443

9. Zhang Q, Pan J, Zhao M-X, Lu Y-Q (2020) Clinical value of the emergency department in screening and diagnosis of COVID19 in China. J Zhejiang Univ-Sci B. https://doi.org/10.1631/jzus. B2010011

Publisher's Note Springer Nature remains neutral with regard to jurisdictional claims in published maps and institutional affiliations. 